12.

\title{
Üher 3etgenheneisanträge im Strafnerfabren.
}

\author{
(Nacdtrag.)
}

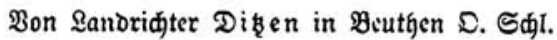

I.

Sherr Reidgsgeridtstat Stenglein bat meinen in Z X 111 bis 167 abgebruđtten 2luffą̧ über ßeugenbemeisanträge im Straf= verfahren eirrer Befpreduung gewürbigt (Daf. S. 475-486), bie allerbings burdineg tabelno ausfällt, bie aber bie Sauptfrage: mann fann ein in ber Sauptverbandlung geítellter Zentgenberveis: antrag abgelebut werben, nur fitreift unb meiner 2 Antwort bezüglich biefer frage weder billigent nod mifbilligento gebentt. Ferr Steng= lein fagt nur:

Buzulaffent ift jeber Beuge, ber audh tur geeigntet ift, bie aus Dent übrigen Beweismitteln gezogene überzentgung

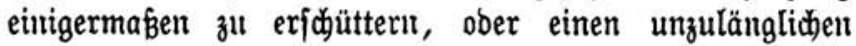
Bemeis zu verítärtent, . . . ęs genügt, menu bie auf bieje Beife berwiefene ober wiberlegte Thatfache auch nur bie geringite ftrafredtllide $\mathfrak{B}$ Birtung zu äukern geeignet ift (S. 479). ")

Sfit bas ridtig, - unb id zmeiffe feinen 2lugenblicf baran, Dás es ridtig ift - jo fino wir über ben Sauptpuntt einig. Dann bürfte auth berjenige Saķ ridutig fein, ben idy per argumentum a contrario bem $\$ 244$ 2tbf. 2 Str.\$.D. entuommen babe utho ber int wefentliden fo lautet:

Jn bem ßerfahrent vor bell Straftammern ufw. . . . be:

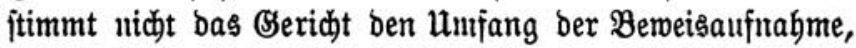

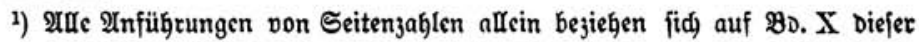
3eitförift.

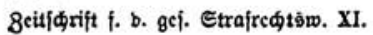


bas. (Serid)t ift bei berjelben vielınebr burd) bie Irnträge ber \$arteien gebunden (S. 121).

Und wenn ber Str.\$.D. Demn ein ßorwurf gentadt merben joll, jo jei es ber, baß̧ ber Bsejezzgeher es unterlafjen hat, biejem Grunojaßze fo ober in äbulidier \$Seije wirflid) 2Ausbrud zu geben.

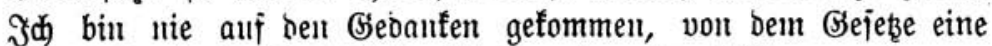
"todbbuchartige" (S. 476) Entid)eioung ber Ëinzelfälle zu verlangen, bie gemís immer lüdenhajt bleiben müßste, uno bei ber zunt minbejten

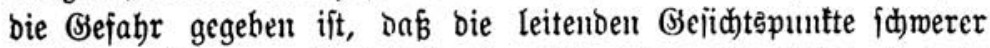

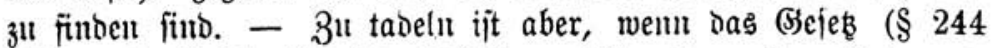
Mbj. 2 Str.\$.D.) für bie Shöf̈engerichte einen Grrundją̧ aus= brüđtid) alşpridut:

Das (jeridjt ift an Anträge nidht gebumben . . .

und wemn ę im unnittelbaren 3ujammenthange banit für bie

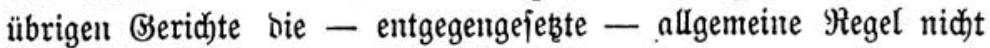
qusjpridft, vielmebr (baj. $\mathfrak{A b j . 1 )}$ nur eine Einzelentidjeioung gibt:

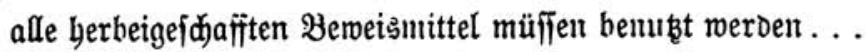

Die Ridjter ber Straffammern unb Sdmurgerid)te, namentlid

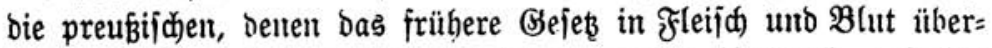
gegangen war, fint meines (Erad)tens gerabe bicrburd) allf eine

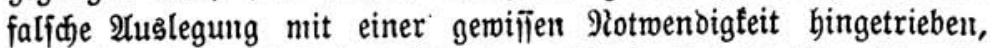

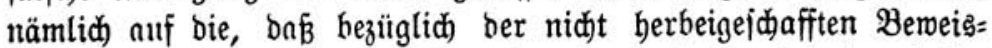
mittel bie Stellung bes Geridhts eine andre, freiere jei, bas beiṕt, ba (Brenzen nidjt erfidstlit) finto, eine ganz freic fei.

Sisobei baun freilid) bas bem (Bejes bireft miberiftrebente

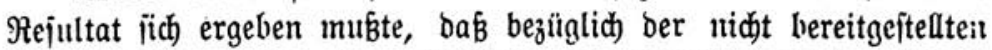
Berveismittel zwiiden belt Ridjtern Der Sdjöffengeridte, Straf= faummern uno હd)wurgerid)te gar feil unteridjieb mebr gemejen märe. Aber jente falfhe Âslegung ift ja aud), foreit id jeben fann, nie in allgemeiner formulierung ausgejprodjen, fonbern immer mur in Einzelfällen thatjäd)lich) zur (B)eitung gefommen, mie

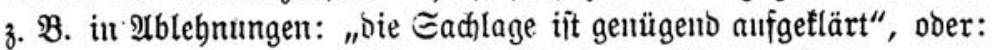
"bein neuen Beugen wiro Das (Berid)t bod) nidht glauben".

Pieine $\mathfrak{A}$ rbeit jollte biejer $\mathfrak{A}$ tslegung entgegenmirfen, und um bies recht beutlid) hervortretent zal lafien, gab id bem meines $\mathrm{Er}$ :

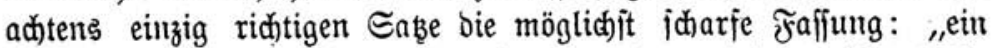
Beugenbemeišantrag faun niemals abgelebnt weroen" (ङ. 120).

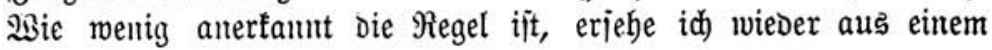




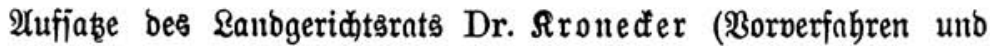
Sauptverfabren (S. 487 ff.), in meldhem auf S. 529 f. zu lejen ift:

"3umeilent eradbtet bas Beridjt ben 2 ntrag nur beshalb

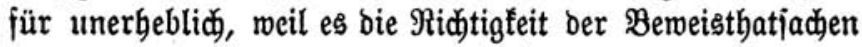
jdyon anbermeit für ermicfent erachtet. Şl ben meiften Fällen ifit ber Bemeisgegenftano zwar ein erbeblider, aber... es exfolgt die 2ublebnung ... unter ber vom Reid)sgerid)t gebilligten Formel, weil die betreffenten Beweije... in Dem ipeziellen Falle gegentüber ben für

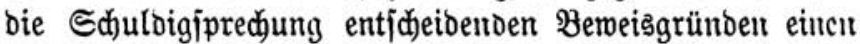
mejentlid)en (sinfluß auf Die \$่emeiswüroigung nid)t üben mürben. ${ }^{2}$ ) In biejer Annabme taun fich das (Şeridjt alleroings aud tăujđen ... weil man bie Erheb= lidjeit eiucs Bemeismittels mit Sidjerbeit erft bann beurteilen fann, wenn man basfelbe vor (i i) hat. Aber weil bies in einzelnen fälllen vorfommen fanu, fo ift bod, fein Brrunb ba, in alfen Fällen bem $\mathfrak{A n}=$

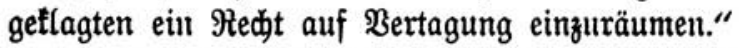

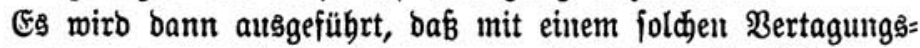

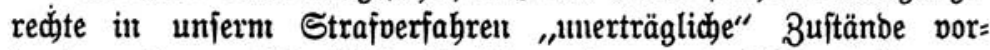
hanben wären. Wir füblen fie fidon oft genug! Aber wir fommen nid)t hinmeg über bell Saß̧, baß̧ bie Erbeblidjeit, bas beiß̧t hier ber Beweismert eines Bemeismittels erît bann beurteilt werden fann ${ }^{3}$ ), menn man es vor fid) bat. Diejer Saz wiro allerjeits anerfaunt, aud von berri Stenglein:

$\left.{ }^{2}\right)$ Serr Dr. Rroneder empfieğt Gierfür in einer Note bie türzere $\mathscr{2} b=$

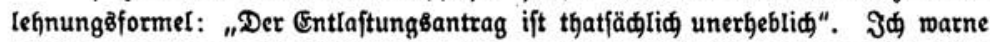
Davor. Die Rronederíde Frormel lann greierlei bebeuten. Erftens: Die unter Beroeis geftellte Thatjade ift (aus redtliden (Srünben) für bie Entideidung uner: heblid. Dann ift bie \&blegnung zuläfifig, falls bie in bent 2lblegnungsbefdluffe zu nennenden oder fonft erjidtliden redtliden Brünbe unanfedtbar find. Das meint aber Serr Dr. Fronecter nidht. Er meint Den zweiten Fall: Die unter

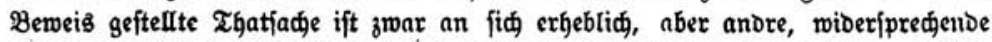
Thatfaden find voll bewiefen, Darum ift ber ganze थntrag unerbeblid. - Diefe Xblegnung ift unftattgaft.

3) So mup Der Sa̧̧ de lege lata heínen. Db aud de lege ferenda ift eine ganz anbre Jrage, bie id abfidttid unerörtert gelaffen habc. \$icr

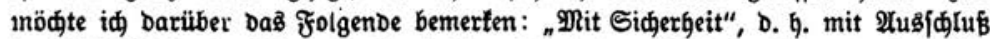

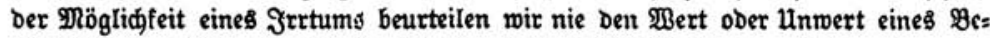

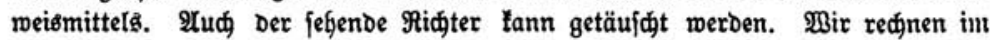

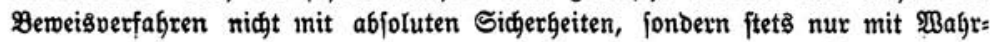


Die Bebeutung eines Seugen läpt fid erft beurteilen, wenn man ihn gefört hat (ङ. 479),

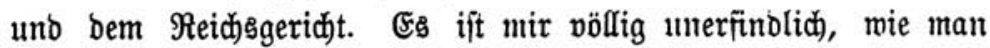
banteben bie Möglidjeit zulaffen famm, baß Beugen beftimmter $\mathfrak{Y}$ rt

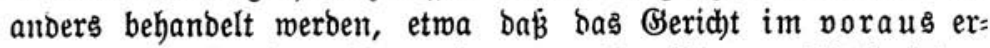
flärt, es werbe einen Bermanotent bes 2lngeflagten nidatt beeibigen uno ihm baun "nidht jo viel Blauben fadenfent, um bie anderweit beglaubigte Thatjadje für wiberlegt zul halten" (S. 479).

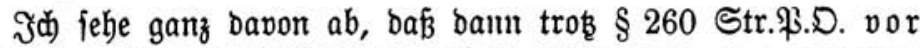
๔d)luß ber \$emeisaufnabme entidieden wirb; von zmei \$ereifen ifjt ber eine erhoben, ber anore nidft; beibe aber merben ge=

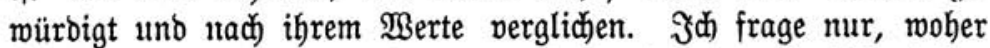

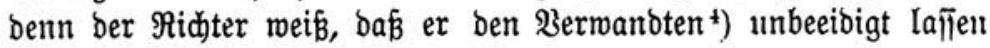
uno ofne şlauben bören wirb, woher fennt 'er bie Meinung des nad) erfolgter Bertagung ber Werhantolung vielleidyt ganz ver:

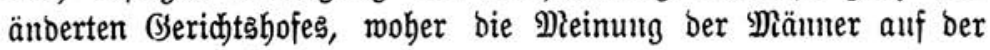
Bejdimornenbant?

Plun fagt Werr Stenglein, jolde bivergierente Entfideibungen

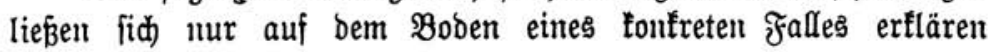

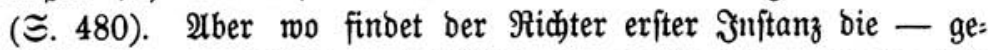

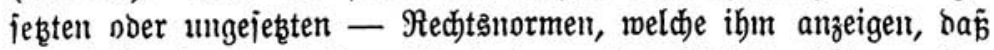

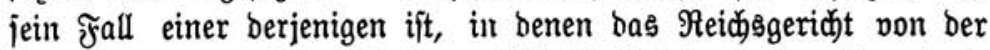

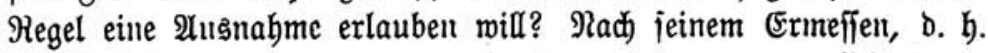
nad) bem nod) jo lebendigen (Šefüble, es wäre unerträglid, wenn hier nod) zur Errhebung eines erfabrungsgemäß minderwertigen $\mathfrak{B} e=$

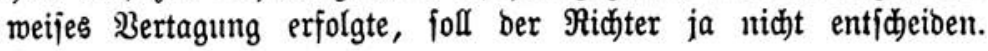
Denn

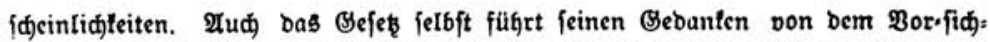

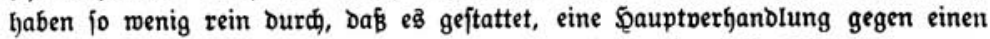

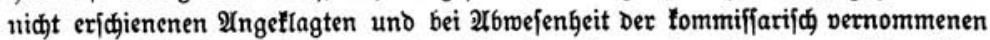

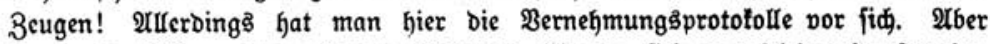

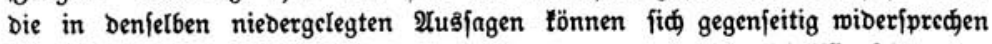

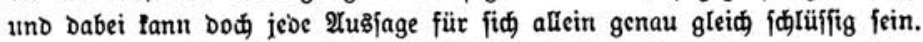

4) Dhne 3reifel würbe Das Reidgsgeriđt aud bei ben Berwandten nidjt ftef̧en bleiben. Siđjer würbe es neben Den in $\S 51$ Str.\$.D. aud Die in $\$ 56$

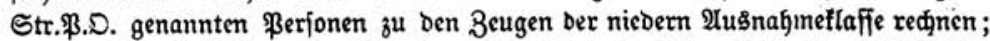
und weiter vielleidt gute Freunde und Feinde? Doer foll die Buläffigteit Der uneiblidjen Bernehmung Die Brenze bilden und meşhalb? Denn bie Be:

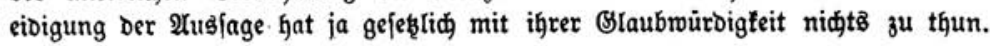


Das (Serid)t bejtimmt ben Uumfang ber Berveisaufinabme nidht nach jeinem Ermefifen; maß̧gebeno fint bie Anträge Der \$arteien.

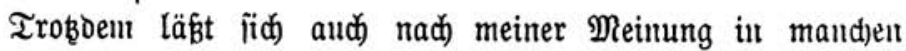
Fällen ber beregten 2 rt zur $\mathfrak{A b l e b n u n g ~ f o m m e n ~ u n d ~ i d ~ b e b a u e r e ~}$

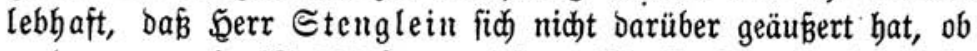
er ben von mir (S. 126 f.) gemiejenen $\mathfrak{B e g}^{5}$ ) für gangbar bält.

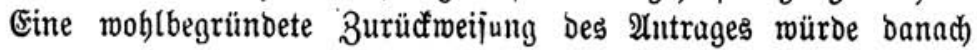
etro lautell fömuen:

Für bie Bemeisthatjad)en haben jo uno jo viele Bettgen gleidjförnity autggejagt; menn ber Angeflagte jeķt eine ihm nabejtebente \$erjon als neuten Siegenzengen in Borjalag bringt, jo thut er bies nidjt in Der $\mathfrak{A b}$ fididt, einen Begent= berveis zu fübren; Das (s)erid)t hat fid überzengt, baß̧ Der Angeflagte antragt.

Dieje Ablehuung erjolgt aljo, weil ber 2nitragiteller nidits be: weijen will, uno nidht, weil er nidats beweijen fann. Das lekstere

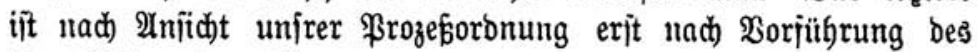
Bemeijes zu beurteilen.

II.

Meine frühern $\mathfrak{A} u s f u ̈ h r u n g e n ~ h a b e ~ i d j ~ b a m i t ~ b e g o n n e n, ~ o a \tilde{B}$ idf mir mein Thema umgrenzte: wie iteht es mit ber Bebanolung

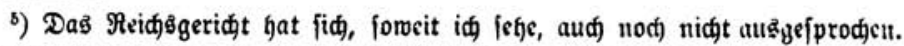

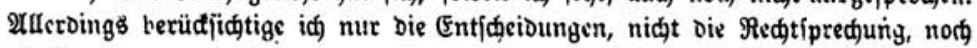
dic Annalen, now Boldammers थrdiv, und berr Stenglein hat mir Daraus

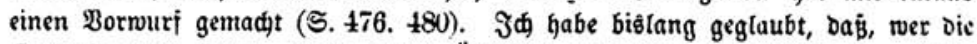

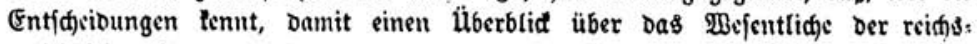
geridtliden Sprudpraris gemonuen hat. Sonft verbient cigentlid dic offitjielle

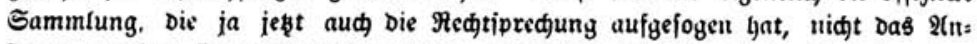

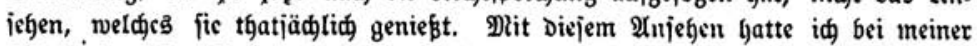

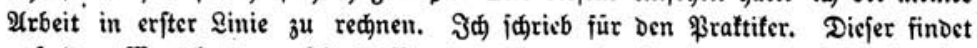
auf Den Wandbrettern fcine B Beratungşjimmers nie oder Dod) faft nie cinc anbre Sammlung als bie Entjdeibungen, und wem id) Die Frage aujwari.

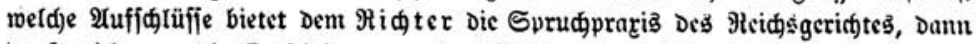

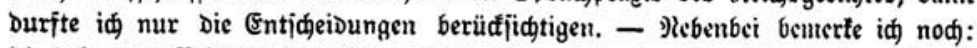

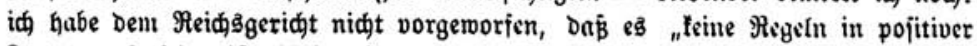

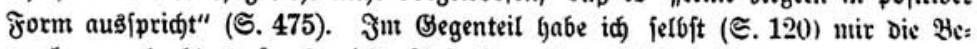

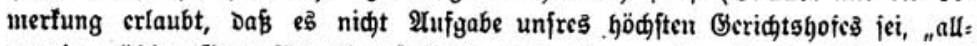

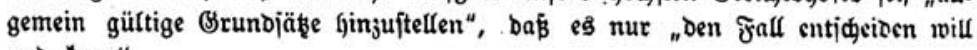
und fann". 


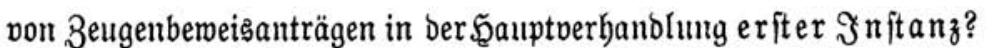
(ङ. 113.) Diejentige frrage alio, weldje ந̌err Stenglein uns it

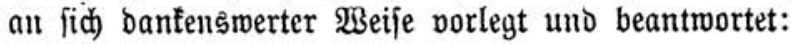

Raun Das Revifions gericht bei ßorliegen eines ungenügeno motivierten, einen Bemeisautrag ablebnenden Dejwlüjes in bie Frage eintreten, ob ein erheblider Antrag porlag, oder muß bas Revifionggericht inegen des prozeflualen Mangels bas urteil aufheben und die Sache ... . in bie Jnitanz zurülđwermeifen? (S. 480.)

- bieje Frage mag vielleid)t "für bie theoretifde Bebandumg" viel midbtiger jein als meil Thema, aber als "Ergäızıng meiner Irbeit in einem wejentlichen \$ulfte" (S. 476), das heißst bod) wohl,

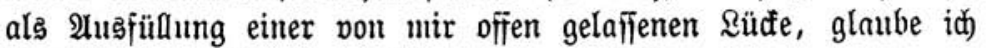
bie Erörterung hierüber nidjt anjeben zu follen.

Dies mebr nebenbei. Bon fachlider Bebeutung icheint mir

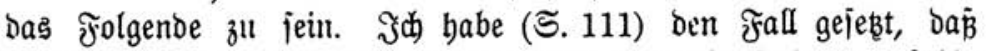

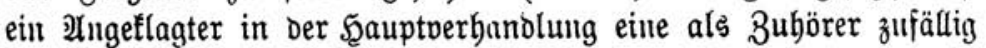

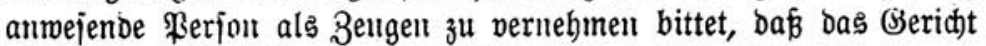
ablehnt, weil ber 3enge nidht gelaben jei, bẩ ber Inngetlagte num bie Raoung und nawherige Bernebunung beantragt, uno id habe baran bie Frage angejdloffen, mann jold) ein 2Untrag abgelebut, wamı nidht abgelebnt weroen fömne. Mohlgemertt, id habe feines=

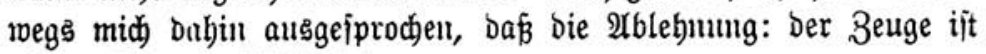
nidjt gelabelt, zuläjîig jei.

Siterzu bemertt Şerr Stenglein:

Der Mangel ber Rabung ift überhaupt fein Brumb, bie Bernebmung eines Zeugen abzulebnen., fondern uur ein Gruno, meldher bas (Seridht berchtigt, bie Erbeblid)teit bes Beugen in Betradtt zu zieben, während bus Borliegen eiller Labung zur Sernehmung Des erfidienenen Zeugen in ber Siegel ${ }^{6}$ ) zmintyt. Die \&abung cines anmejenten Zeugen braudit gleidffalls nidjt mebr bejolofien zut merben, uno nie hat bas Reidsggerid)t einem fo geijtlojen Formalismus gebuldigt, Daß́ es forberte, es müifie bem vor Dem (S)ericjte ftebenden Zeugen von Dem Bserid)tspollzieher mitgeteilt werben, er folle vor bem (S̈erid)te erfideinen and bies fei

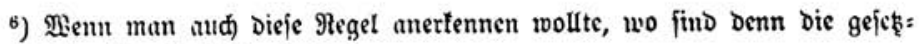
lid,en Bründe für sic 2tus̆nahmen? 
Sedingung ber Sernehmung. Das Gericht hätte aljo nie

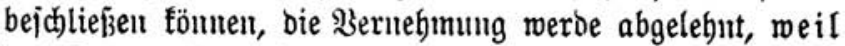
ber 3euge nidht gelaben jei (ङ. 476 f.).

Mit bent leb̧tell Są̧e bit id volffontmen einveritandent uno ich habe, weum aud) viell(cid)t nid)t ganz beutlid), nid)ts anbres gejagt. Jebenfalls babe id) nidhte anbres jagen wollen. Son meinem Stantopuntte aus fonnte idh gar nidjt Darauf fommeni, ben $\mathfrak{X b}=$

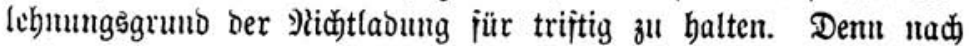

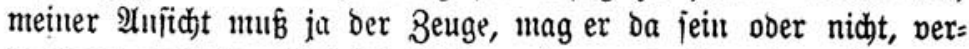
nommen merben. $\mathfrak{A}$ uf bie \&abung funn es aljo nie anfomment.

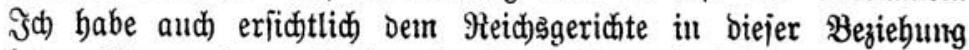

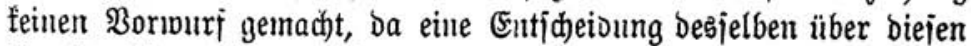
Funit nicht vorbanden ijt.

Wir finto alio einig: es müroe "geijtlojer Formalișmus" jein, ment man bie Rabung Der anwejentent Zeutgent als, ,Bebingung ber

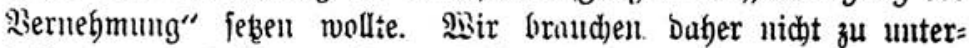
juchen, menurgleid) der $\$ 244$ $\mathscr{2} b j .1$ Str.\$.D. wörtlic() uur von "vorgel abenen" Beugen iprid)t, ob bie auwejenbe \$erjon nach

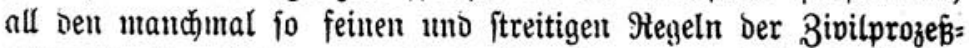
oromung über sabungen citiert ijt. Es genügt, bá bie ßerjon gegentwärtig jei.

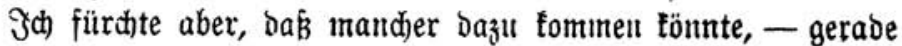

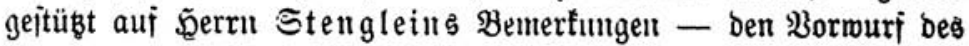

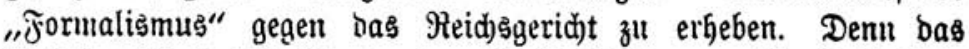

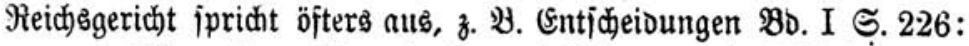
Die erit nadber (D. h. nad) Laoung und Erjdeinen Des 3eugent) bigründete überzeuguıg von Der Unerbeblid)teit Des Bengniijes (๖. h. Der Thatjadje, über Die nusgejagt

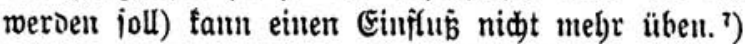

Dies beib́t alfo: ment ber Z3euge nid)t oder, was bem gleid)= iteht, nicht unter genaueiter Şcobad)tung Der zivilprozejīualent Pregelu gelaben wurde, baut muf bie Siernefunungsthatjadje er= heblich fein. Šit ber Zenge aber oromungsmuäß̉ig geladen, Daun hat bas (Berid)t bie Thatjad)e, weldje Der 3euge befunden joul, nid)t mebr auf ihre Erbeblid)teit ful prïjelr.

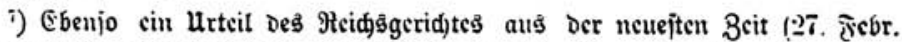

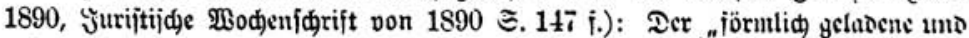

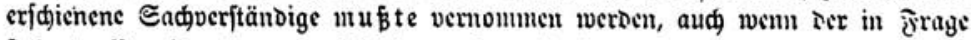

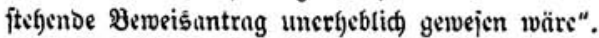




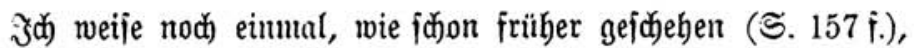
Darauf bin, baß́ es nad) biejer 2nfifid)t gar nid)t notmentig er= ja)eint, von bem 2 Ingeflagten bezüglid feiner felbitgeladenen Bengen bie Ingabe eines ipeziellen Semeisthemas zu verlangen. $\mathfrak{X} u=$ gemeines Bereisthema ift ja ber in bem Eröfinumgsbejdlulfie be=

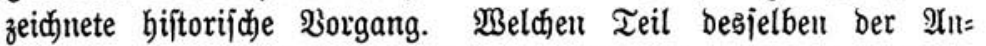
geflagte gerabe bei biejem Beugent im $\mathfrak{A}$ uge hat, ift gleidgültig. Denn jelbit menu biejer Teil unerbeblid fein jollte, fo muß ja bie Sernehmung exfolgen - eben wegen ber Rabung.

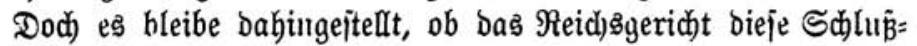

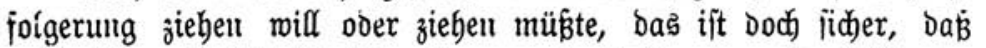
nach feiner 2 Anfid)t bie \$ernebmung eitres Zeugen und, falls eine Beruebmung abgelebut murbe, oas Sdjidjal eimer Revijion bavon abbängen fönnte, ob Der Zettge oromungsmäßig geladen iit, z. $\mathfrak{B}$. ob ber Poftbote Den Bororud jeines Buiteflungsbogens ridutig burd)= jtrichen hat.

$\mathfrak{A l j o}$ märe bie Rabung Dođ) Worauşę̧ung ber Bernebmung uno ber Formalismmı wäre da? Er ift allerbings ba, aber bie Sd)ulo liegt in Gejeke. Denn unbeitreitbar ift, baß $\$ 244$ 2bi. 1 Str.ß.D. (agt: id) bin anmenbbar auf bie "vorgelabentu" Zeugent. Frage ift nur, mas bies bedeutet.

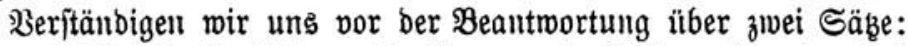

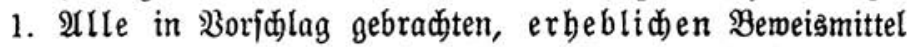
müfien erboben merben (pgl. oben S. 461 fi.). Someit Bengen in Betradjt tommen, madjt es feinen unterjajied, ob fie anmejend jind, ob nicht, und wenn fie anmejent find, ob fie eine Sabung vom Beridhts volfzieber erbalten haben, ob nidjt.

2. Rein als unerbeblid) erfantes Bemeismittel ijt zu er=

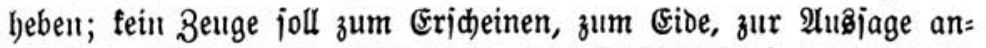
gehalten, nötigenjalls burd) (ङelD= uno Freiheitsftrafen gezmungen

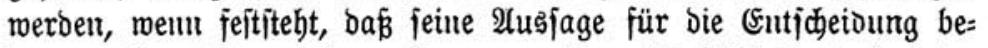
Deutungslos fein mürbe. Genauer: ebe nidjt feftiteht, baß jeine Anşage

"ein Ergebnis als möglid in भueficht jtellt, weld)es ge: eignet erid)eint, bas Bsejamtergebnis bes \$emeisperfabrents irgenowie zu beemflufijen" (Stengleiu S. 477 f.). ${ }^{8}$ )

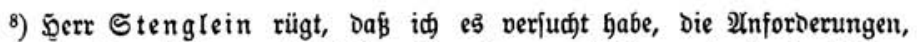
dic man an cinen joldjen erfebliden Bemeisantrag ftellen muß, in ein Snf́tem 
Das folgt unmeigerlich aus bem 3 wede ber Berveisaufuabme: Aujtlärung Der Sadje, uno Daraus, bẩ bie \$flid)t Des Zeltgen nur

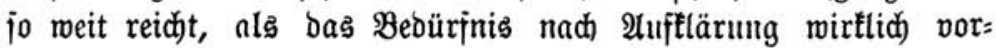

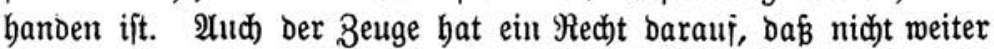
gegangen merie. Was nidjt zur Sadje gebört, gebört nid)t in die Berbandlung. Ehe nidjt ein Berveisthema genaunt ijt, faum gar nicht die Frage entitehen, ob ber $\mathfrak{A b m e j e n d e ~ g e l a b e n , ~ o b ~ b e r ~} \mathfrak{A n}=$ mejende vorgerufen und zum Erbeben ber Sdyurbanto aufgejorbert werden joll. Wollte man bies nidbt anterfennen, fo würbe mant bie Srrenzen einer Bemeisaujnabme überjobreiten und ins Srrenzenloje tomment, wo auch eine Unteridjeidung zmifden "unerbeblid" uno

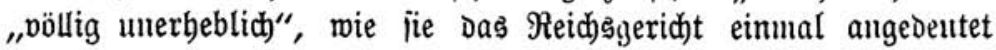
bat $\left.{ }^{9}\right)$, nidbt meiterbelfen tönnte.

\$enu mir biefe Säke anertennen unb von ihnen ausgeben, jo folgt:

a) alld ber auf Rabung aumejende Beuge ift mur über Er= hebliches zll vernebmen; bie Benenmung einer Ihatjache ift aljo notmentig; menn bie benaunte Thatjache unerbeblich ijt, jo miro ber Beuge nidft vernommen. Dies fagt uns Saß̧ 2 (oben) und es

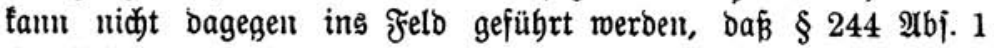

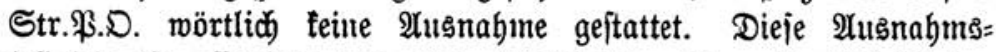
lojigteit ift offenbar mur eine fd)einbare. Dhne Zweifel bürfen

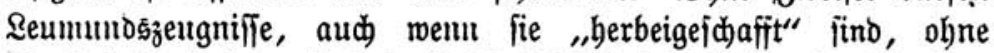
3meifel barf bas "herbeigejdaffte" \$olizeiprotofoll über bie :er= nehmung eines inzmijhen veritorbenen Beugen nidjt verlejen werden, uno ebenjomenig follte es bezmeifelt werben, ons bie $\mathfrak{A}_{\text {usfagepflicht }}$

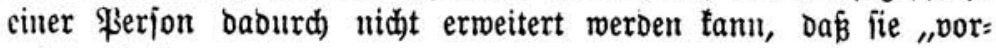
geladen" ift.

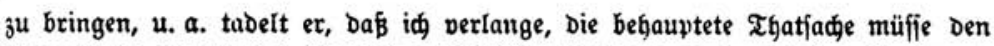
Begenftand gerade Des Zeugenbemeifes bilden tönnen, und er meint, Die Beant:

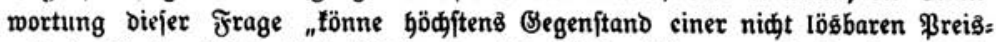

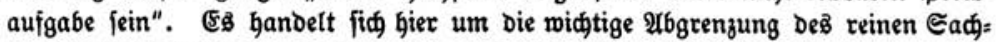
verftändigenbemeifes gegenüber Dem \$eweife Durd fadjverftändige Zeugen, Die

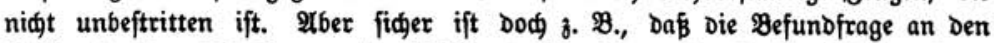
Seftionsarzt, meld)e Drgane Der Stid getroffen yat, reine Sacjverftändigenfrage

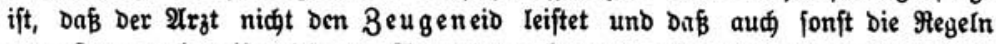

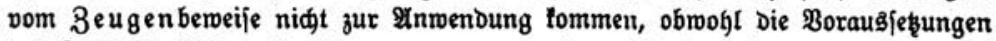
Desి § 85 Str.\$.D. wörtlid) genommen vorzuliegen ideinen (vgl. aud S. 162 ff.)

9) Entiđ. :Do. I S. 244. 
b) § 244 Abj. 1 Str.\$.D. fann nidyt jagen mollen, baß alle aumejenden vorgelabenen Beugen vernommen merden müjīt.

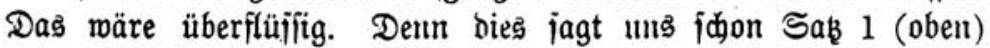
für alle Bengen foblechtweg. Das Bejeb will hier für bie vor= gelabenen ${ }^{10}$ ) Zenigen etwas 2 ntores, Bejonderes vorjdreiben.

Bas ijt Das. Bejondere? Es liegt in ben Borten:

"ßon ber Erhebung einzelner Bemeije fami jebod) $a \mathfrak{b}=$ gejeben werben, mem bie Stantsanmaltidjaft unb. ber $\mathfrak{A} n=$ geflagte hiermit einverítanden jino."

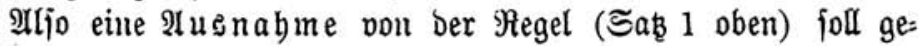
jđaffen merden, und bieje Ausnahme liegt nur vor, ment bie \$arteien, mie id) früber bereits gejagt habe (ङ. 121), auf bie $\mathfrak{B e r}=$

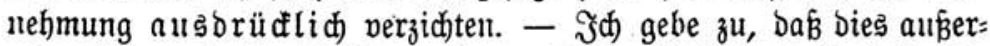

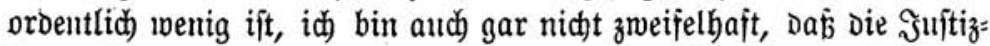
tommiffion, non der bie Bejtinmmung bes $\mathfrak{A b j} .1$ \$ 244 Str.\$. ftammt, Damit fehr viel mehr hat jagen mollen. Sie bat uns

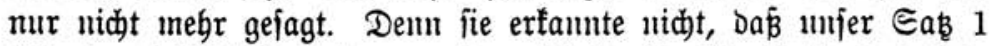
(oben) uıbejdränft gelten mußste, fobalo bie Boridrift Des Ent= murfes (§ 207 Daj.):

Den Umjang Der Berveisaujuabme bejtimmt bas Geridjt . . . von igr gejtrid)en, bez̧iebungsmmeije auf bie Schöffengerid)te bej(bräntt worben mar.

unto man ijt jeşt boch wohl einig, Dẩ nur basjenige ent:

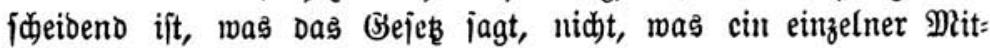
arbeiter an SBejek hat jagen mollen.

\section{III.}

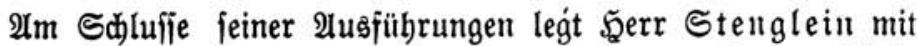
bejonderer Betonung ernite Berwabrung ein gegen bie angebliçse

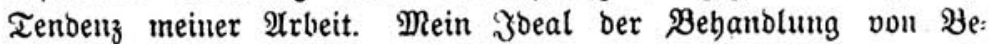
meiłanträgen jei die "Sd)ranfenlofigfeit", die "Souveränität des Thatridyters in Der Bebandung Der Frage, melche Beweife er ent:

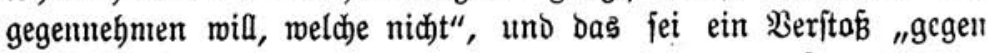

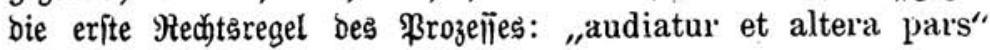
(ङ. 482 f.).

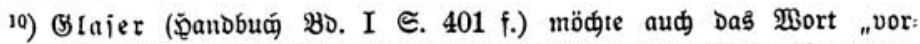

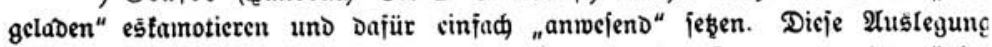

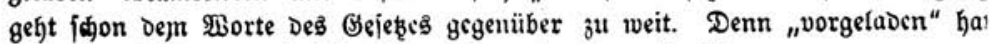
eine ganz bef́timmte tednijळe Bedeutung neben "anivefend”. 


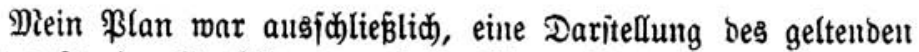

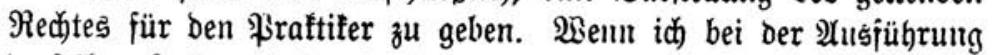
Desfelben fand und in Fortidgreiten ber $\mathfrak{A r b e i t}$ immer mebr füblte, dá̧ Der von unjerer Strafprozéboromung geidaffene Bodent

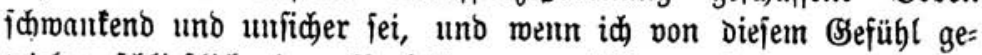
trieben ichließslich einen Berbejierungsvoridilag mebr mur andeutete, fo dürfte bies faum Tendenz fein, "pon ber id bei meinen Dar:

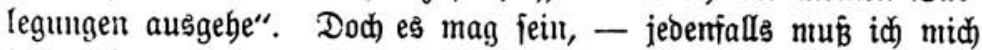
jekt auf ben erbobenen Borwurf hit etwas näber mit ber Sadje bejäjätigen.

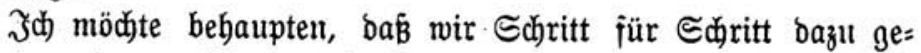
bränģt merben, nid)t mur bie Bereismüroigung, foubern auch bic Entideibung über ben $\mathfrak{u m f a n g}$ Der Bemeisaufnahme bem ridjter= lident Ermefien zu überlaffert. Spridht es bod jelbit jekt, mo bas

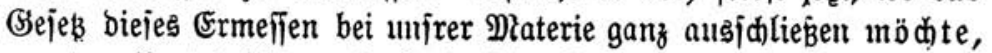
wo der \$arteimille maß̧gebeno fein foll, troß̧ allebem in einem Sauptpuntte bas enticheibende 20 ort, nämlich bei Beantwortung ber itets zu erbebenden Borfrage, ob Der geitellte $\mathfrak{A n t r a g}_{\text {wirflid eit }}$ Berweisantrag jei. Die Antwort hierauf muß́ foiort gefunden merDen, ein Sinausjojieben bis zum $\mathfrak{u r t e i l}$ ijt hanogreiflid unntöglid

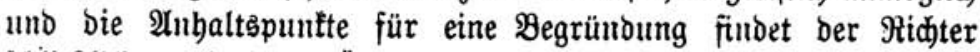
¡đblię̧lich nur in jeiner überzenguıg. Naturam expellas furca! . .

Ruı meint Gerr Stenglein:

Mas ift bas für ein G̈ebör, wenn ber $\mathscr{A}$ ngetlagte żmar $\mathfrak{B e}=$ liebiges behaupten, aber aud) Das \$ejentlide nidjt be: meijen Darf, went es Dem SRid)ter nicht beliebt?" (ङ. 483.)

Dies mürbe von (sjervidjt, vielleidit von Ssemidht fein, wenn es

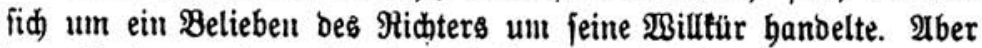
bas ift ja gerabe. nidjt ber Fall. Der Stidjter foll hier nidjt "founerän", nidht "idurautenlog" jein. Der Souverän ift bas

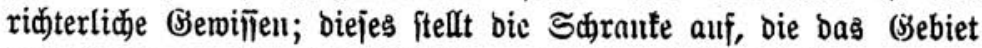
ber Pflidjt von bem ber Willtitr zu fdjeioen beitimmt ift.

(E) hat eine Zeit gegeben, in Der bie Bjejeßggeber verjudten, burd) Anfitellung von Bemeisregeln bie Bereismittel gleidjam zu eidjen und mit eiuem Bemidjtstempel zu verjehen. Wenn ein

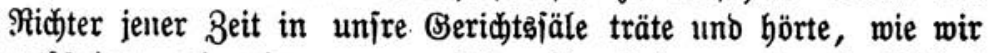
entidbiden, mie wir - unr mit ber Begrünoung: "Das (Serid)t hat iid) überzengt" - mandjmal bem beeibigten Bengen A gar nidjts, 
Dem unbeeioigten Zeungen B alles glauben, er mürbe fidjer, went er vor Erítauntel oder Ėntriifitung bas Wort fäntoe, jragen:

\$as iit Das für ein Bereisred)t, wenu es Dem 2Al= geflagten geitattet ift, alles zu bemeijen, Dann aber ber

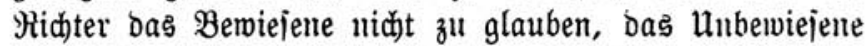
zu glauben bejugt ift, wie es ibm belieht?"

Der Rormurf mürbe, weun bort, jo hier gelten; in gleidjer Weije aber audi) mein Einmand: es beliebt nidjt!

Das Snjtem ber freient Bemeismüroigung ift ja eben aud) nidjt

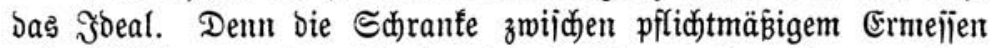
und \$illtïr, von ber id) oben iprad), ijt zmar ba, aber zll zeigen

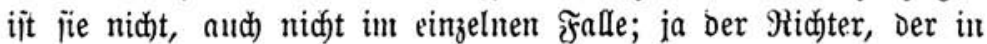
peinlichifter Treue zu enticheiben beitrebt ijt, wiro oft empïntoen,

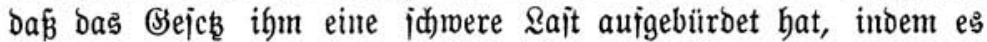

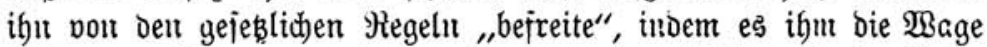

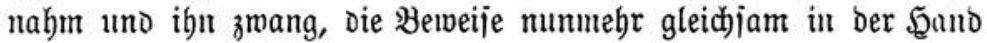
nach jeirem (bsefühl zll wägen.

Yrber man hatte mun einmal eingejehen, Daf jede 2 Bage bisher unzulänglid) uno faljà) mar. Darum marf man fie zur Eeite uno gab bie vergebliche $\mathfrak{A r b e i t}$ bes Sudjens auj. Man nahut das Beijere an Stelle bes unerreidjbaren Beftelt.

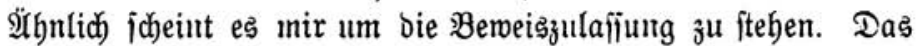

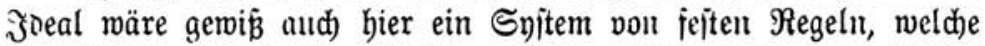
einerjeits gentïgende ફantobabe gemähren müßten, alle gegen iträfe Durdführumg des Strafperfabrens geriduteten Beftrebungen it Sd)ad) zu balten, anorerjeits aber bie beredtigten Jnterefien Der

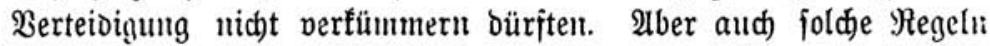
fino unjinobar. Darum ijt mit ibuen nid)t zu red)nen. Daun aber bleiben żnei Diögliwheiten: entweoer es entid)eibet bie \$artei over es entidbeibet oer Ridjter. ") Die erite Alternative bat idjou

11) Ės gäbe wohl nod) cinen Dritten, mittlern $\mathfrak{B e g}$ : Die berciten Beweisz=

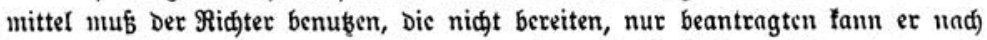

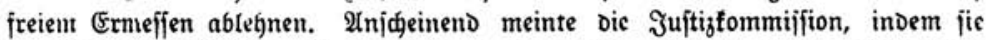
Den $\mathscr{A b j} 1$ Deș $\$ 244$ in Die Str.\$.D. einfügtc, Dicjel: Weg zu gefen. Wenigítens wird bit Den Beratungen mehrfach erörtert, wie Das (seridgt fich vor einer nturba

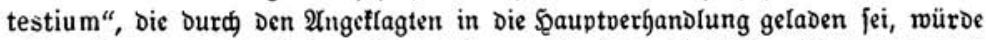

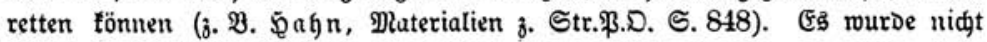

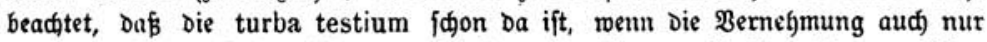

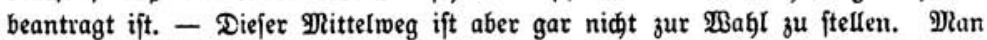
würde ein \$rivilegium für Den Staatsanwalt jळaffen, Der ftets 3eugen Iaden faun, wägrend Der regelmäßig unbemittelte 2Ingetlagte eș nidjt fann. 
voll vornberein eit :Bebentliches. Man fann bie \$artei, o. h. ben Ingeflagten nidht bitton; bei igm mürbe bie Entideidung aufs reime Belieben gejtellt fein - und jo ift es jekst! - er mürbe fid nur von jeinem Strebeli nach Freijpred)ung ober mildeiter Strafe

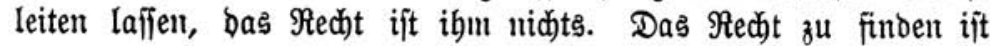
bem Ridjter afles, er fum an jeinen Eib und an feine Pfflidbt ge= bunben werben, und in jeiner ફ̧ano rubt aljo jójon nad) biejer Er:

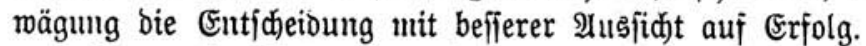

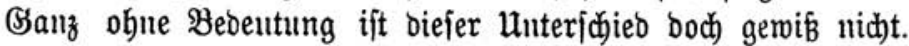

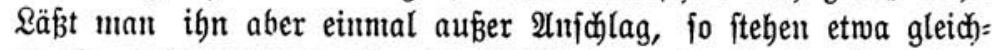
wertig nebeneinanoer hier bie Entjockioung burd) Die ßartei -

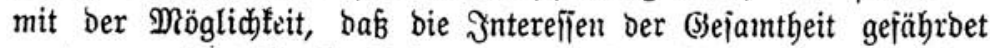
merben - Dort bie Entjdjeioung Durd) Den Ridter, mo zmeifellos in ähnlid)er Weije, namentlich bei jebr überlajteten (S)eriduten, bie

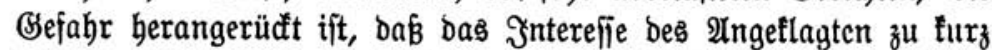

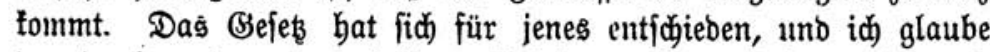
im einzelnen in meiner frübern 2 rbeit gezeigt zu babelt, wie grof bie Utnzuträgliç)feiten finı, bie baburch veruriad)t merben fönuten.

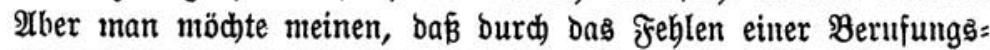
initanz und burch bie freie $\mathfrak{B e m e i s m u ̈ r b i g u n g ~ b e r ~ A n g e f l a g t e ~ v o n ~}$ vorberein benadjteiligt merbe; es jei baher nur geredt, wenn man ihm zun Entgelt bafür bie foutveräne Mađbt über ben Umiang ber Bemeisaufnabme verleibe, banit ex bieje Dadjt gleidjam als Sidjerunggoventil benüßzen fönue. Dieje (Ermägung bietet mir alth)

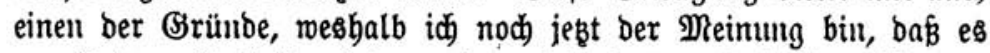
zur Zeit nod beffer fet, an bem bejtehenden Buitanbe nidits zu

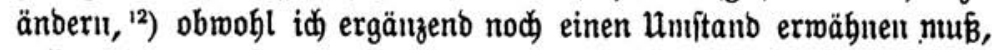
beffen Bebentlidfeit jeber nur einigerınaisen Sadflunoige fühlt, obne baß̧ er barum gerabe bäufig, zumal in biejem B̧ujummenbange, zutr Sprade gebradt miro.

Man weißß, wieviel Einfluß̧ ein verftänbiger, Lonaler ßer= teibiger auf bett Bang bes $\mathfrak{B}$ erfabrens bat. $\mathfrak{B O}_{0}$ er wirft, fomment

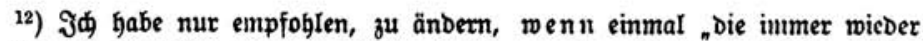
auftaudenden Beutühungen um \$iedercinfügrung Der Berufung Erfolg haben

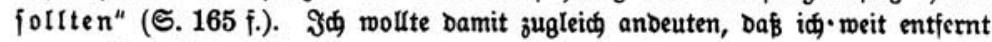

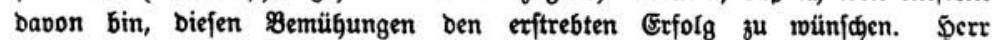
Stenglein fdeint mich bier mifberftanden zu haben, indem er fagt, id wolle nDie von mir verlangte (?) Reform in Berbindung mit Misedercinfübrung Der Berufung bringen" (ङ. 484). 
jelten überflïijitge Bemeisanträge unb alle Sdjwierigfeiten fint ge= hoben. Das bedarf feiner 2tugfübrung. Mun brängen fid aber

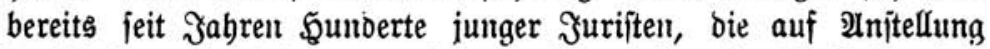

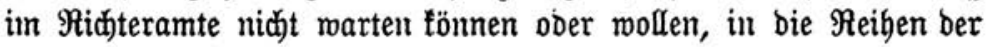
Inwälte, uno man hat wohl nidjt ogne eine gemiffe Beredtigung bie Befürobtung laut merben laffelt, Daß̧ im Rampfe ums Dajeit aflmählid) ein Inmaltôproletariat beranmadjien tömue. In ber ظand eines fojlediten Inmalts aber, id) meine eines ßerteidigers, jür ben feine Praris in erfter Sinie, jein Rlient in zmeiter Sinie

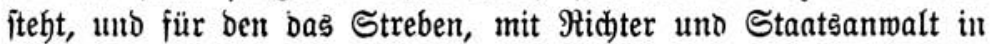

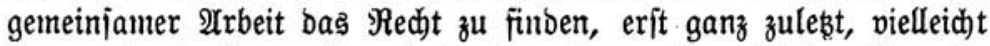
jogar nie in Frage fommt, in ber ছanto eines joldhen Inmalte wäre bas heutige Bemeișredt eine furd)tbare Sd)uz̨mafie gegenüber bem Angriffe Der Strafoerfolgungsbehörocit.

Ginterthüren für argliftige \$rozekfiührung hat mohl bielang nod) jebes Beję gezeigt; bier aber liegen fie gar zu bequem und verloctento!

Das fino bis hierber nur theoretijde Ermägunţen. Bom praftijden Stanopuntte alts babe id geltend gemadt (ङ. 166), Daß̄

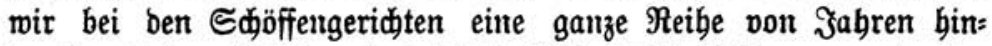
burd) mit ber $\mathfrak{B}$ irtung bes $\S 244$ 2hbj. 2 Str.\$.D. nur gute Er= fabrungen gemadt haben. Werr Stenglein meint (S. 483), man

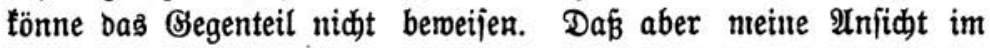
Stidteritanto bie meitaus vorberridjente ijt, bafür barf id mich mohl auf bie Serbanblungen bes 18. Juriftentages ${ }^{13}$ ) berufen; id fum mid aber aud bafür nod) auf eine andre Thatjadje bezieben, beren Ridtigteit mir meine Rollegen aus bem Ridteritanto bes ftätigen werden, nämlid) auf bic, baß̧ bas fog. freie (Ermeffen bei

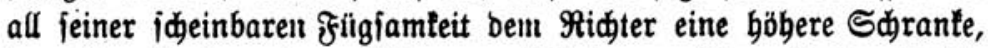
Dem Angetlagten ein wirljamerer Sduz zu jein pflegt, als bie mehr ober mentiger ftarre uno fpröbe geję̧licje Regel, oaß z. $\mathfrak{B}$.

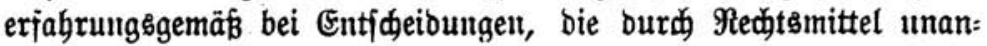
greifbar find (4), bie Sadjunterjudjung und Begrünbung im (5)efüble ber gejteigerten Berantwortlichteit eine gallz bejonders eingebentore

33) Bgl. aud Z VII 250 ff.

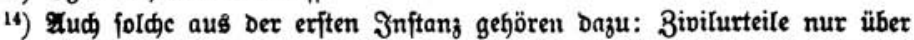
Soften u. Dergl. - Ramentlid ift mir ber im Tert angefügrte umftand früher in Sannover aufgefallen bei ben nad Der bannoverfden bürgerliden \$rozeb=

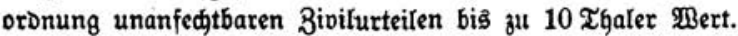


uno forgfältige zu iein pflegt. Es ift mahr, altd) bies läß̈t fid) nid)t berveiien. Doch id) bente, id barf fürs exjte babei bleiben,

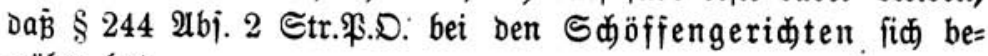
mährt hat.

S'egen jebe Folgerung bieraus verwabrt fid aber. Werr Steng= lein burch bell 3ują̧:

... in bisberigen Umfang Der Inmentoung. Daß man in Straffammerjadjen eine gute Erjahrung nidt machen

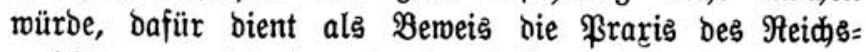
geridjts... bejonders in Den beiben eriten Bänben ber

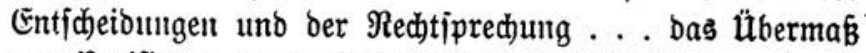
von Revijionen megen $\mathfrak{B e j d ) r a ̈ n t u n g ~ b e r ~ B e r t e i b i g u n g ~ b u r d ~}$ Ableb̆un!y von Bemeisanträgen . . . (S. 483).

Darauf babe iđ) zunädjit zu fragen, was beun eigentlich bie Fraris des Reidjsgeridtes aus ben erften 3abren nad 1879 be= meijent faun? Dod) gewißs nidjts anores alș bies: bie Straf= fontmern verítantoen die Strajprozésorbnung, wie fie jeşt ift, falíd. Sie bliebelt bei ber - zumal in \$reuken - altgewohnten $\mathfrak{A b}=$ lchuming von Bemeisonnträgen nad) (mehr ober melliger) freiem $\mathrm{Er}=$ meīen ftebgen, umb Serr Stenglein zmeifelt mohl aud bezügliç

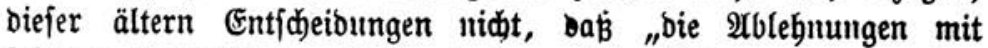

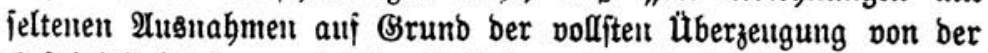
Erfolglofigfeit Der verjuchten Entlaftungsbemeije bejhloffen" wurbert (ङ. 483). Das Reidjggerid)t batte in biejer Ridjtung bie $\mathfrak{A b}=$ lebnungen nidht zlt prüfen; eछ hatte nur zu fragen, ob ber $a b=$ gelebnte Beuge ridatig werben tonnte, uno zur Raffation genügte

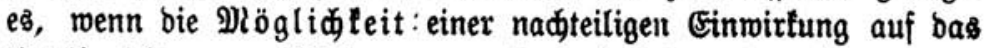

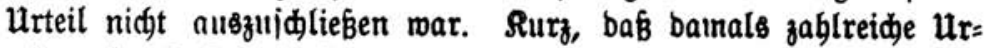
teile eriter Эnitauz aufgeboben unb bie Sadent baun zurüđgemiejen wurben, bemeift nidt etwa, baß bie urteile materiell faljij maren, bấ bie 2lngeflagten zu unredt verurteilt (ober freigejproden) find, fondern nur, baß̧ bie Berveisaufnabmen nidht fo volltändig ftattgefundent hattell, wie bie \$rozézorbnung bies verlautgt. Selbft wenn - was nidjt gejdeben - feftzuftellen wäre, bá̉ it jenen Sadent bie Urteile nad) Wieberbolung ber \$auptperbandung exiter Jnitaliz veränbert finb, fo würbe bies bod) nidjt oḅne weiteres

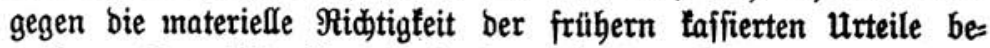
weijen. Denn fein Bemeismittel veriळledtert fid. burd) Beitablauf fo idnell und fider wie ber Zeugenberveis. Wurbe alfo bas Enb= 
ergebnis nach ber Revifion ein anores, fo bleibt immer nod) offene

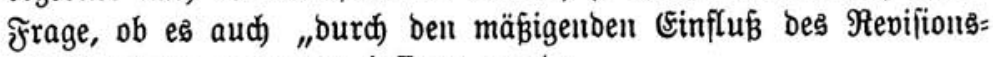
geridutes" (S. 483) ein befieres murbe.

Es jreut mid) aber bei allebem, baß̧ berr Stenglein ben Grund, meshalb $\S 244$ 2 bj. 2 St.\$3.D. bei bell Straffammeru

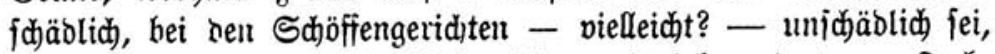
nidjt barin fudjt, baß̧ hier \&aienridhter mitwirfen, bort nur Fact)= juriiten entidheioen. Darum fan! idj es mir mohl eriparen, auf biejen Unteriobied hier eilıugehen. Serr Stenglein finbet Den

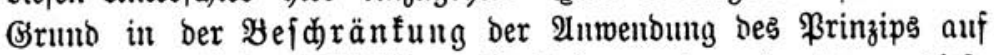
bie minder midjtigen Sdjöfïengerid)tsjachen uno er beutet an, Dieje

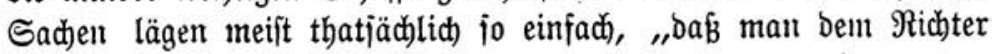
nid)t zumuten töıme, eine grö̈ere $\mathfrak{A} n z a b l$ von Zeugen abzubören". (ङ. 483.)

গun find bie thatjächlid) einfachen Sadjen im wejentlidjen bie übertretungsjachen. Sier tonment meift umfangreidjere Bemeisauf= nabmen und bemgemäß Parteiauträge auf Bernebmung meiterer 3enten nidjt vor, und die Frage, ob bas (S)eridjt von feimer Madjt= befugnis folden 2 nträgen gegenüber (Bebrauld machen joll, wiro gar nidjt auftnuden. . Sleiben bie Bergebensfachent. Jeber weí̈, Daß gerabe bie midtigíten unter ibnen geborne Straftammerjachen find, welde auf Antrag bes Staatsanwaltes bem Sdjoffentyeridbte überwiejen werben fönnen uno thatjädlid immer überwiejen meroell, weltn eben ber $\mathfrak{A}$ ntrag erfolgt. Borausiję̧ung bierfür ifit aber nidjt etwa ber geringere ober größere umfang ber zu er= wartenden Bemeisaufuahnte, fondern bie von belu Staatsantwalte

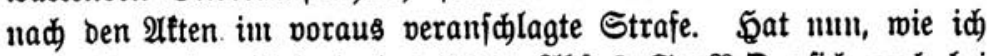

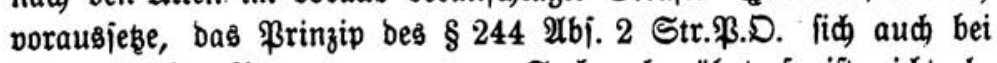
biejen ber Straftammer entzogenen Saden beroübrt, fo ift nidt $a b=$ zujeben, warum es Sdjaben ftiftell follte bei ben übrigen ber Straffammer verbleibenden Sadien ${ }^{15}$ ).

ङierzu fommt, baß̧ injolge ber Beftimmung im $\S 23$ Abj. 3 Str.\$.D. minbeftens brei, aljo bie $\mathfrak{D e b r z a b l ~ b e r ~ f u ̈ n f ~ \Re i d j t e r ~ D e r ~}$ ertenuenden Straftammer bem zu beurteilenden Falle unbefangener

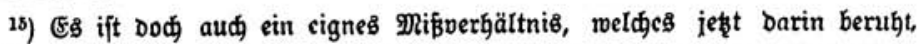

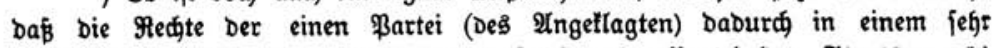

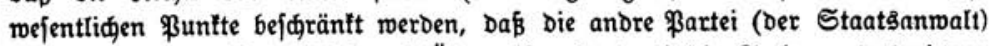
burd eine unangreifbare Plabregel (übermeifungäantrag) bie Sade an basి nieder: Beridit bringt. 
geçenüberjtehelt als ber Sorfingende bes Sdöffengerid)ts, Der bie

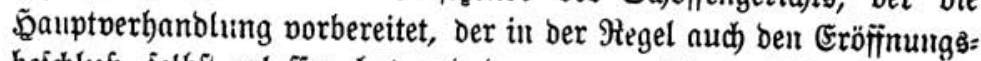

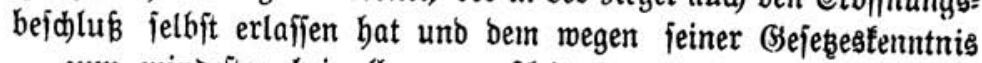
- zum mindeiten bei allen prozeßleitenden Entideibungen - bie Ed)öffen lediglich) beizuitimmen pflegen.

Damit glaube id) berwiefen zu haben, baß̧ eine 2usbebnung

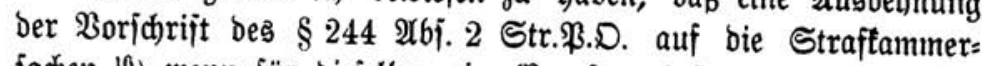
iad)en, ${ }^{10}$ ) menn für biejelben eine SBerufungsinftanz nen eingeridjtet wirb, red)t wohl empfohlen merben taut, uno baß̧ bie von Gerrn Stenglein bagegen erhobetren Bebenten, joweit fid bergleidjen von vornberein überjehen läßt, fic) als hinfällig baritellen. Unto

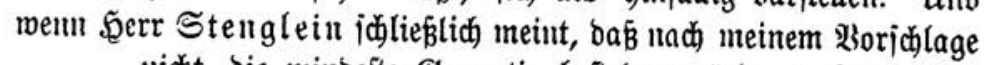
nidjt bie minbefte Barantie heftehen würoe, Daß̧ ber $\mathfrak{A n}=$ geflagte in zmeiter Şntanz mebr Sebör finbet als in eriter... (S. 484),

jo faum von Barantie, o. h. von abjoluter sidjerbeit bes Erfolges einer gejeßzlichen \$orjdrift mobl nie gefprod)en merben, ba bas

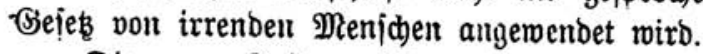

Die neue Jnitanz bringt aber bem Inngetlagten unmeigerlida) zroei $\mathfrak{B o r t e i l e . ~ E r ~ h a t ~ f e i t ~ U r t e i l ~ e r i t e r ~ J n f t a n t ; ~ e r ~ w e i f ~ n u m m e b r , ~}$ was er früber vielleidyt nidjt mufte, woraulf es anfommt, unb er taun, melut es $\mathrm{ibm}$ überbaupt baran liegt, jachlid zu verfabren, jachlidje 2 nträge ftellen uno fie fadjlid begrïmbent. Dazu bas

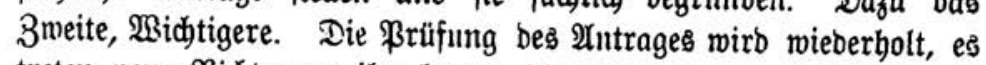

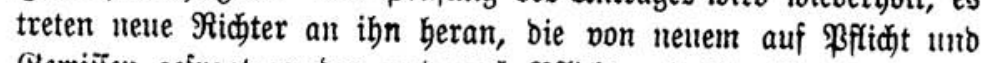
(3)emifien gefragt werben und nad) Pflid)t und Bemiffen autworten werden.

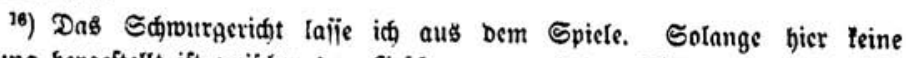

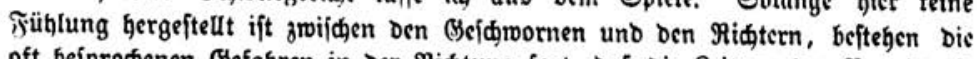

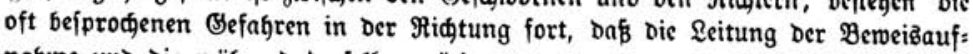
nahme und die wägrend Derfelben nötig werbenden Entideibungen nur von Dem

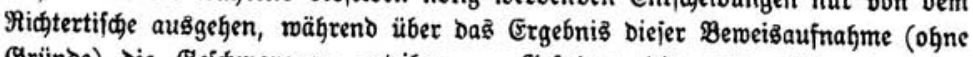
Sründe) Die (Sej申morenen urteilen. - Ecfagren bie nur geiteigert werben

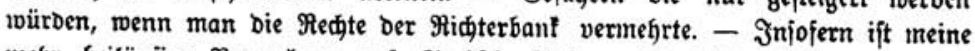

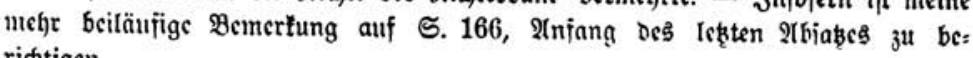
ridtigen. 